\title{
Thermal entanglement in a two-spin-qutrit system under a nonuniform external magnetic field
}

\author{
Guo-Feng Zhang*† \\ State Key Laboratory for Superlattices and Microstructures, Institute of Semiconductors, \\ Chinese Academy of Sciences, P. O. Box 912, Beijing 100083, People's Republic of China \\ Shu-Shen Li \\ CCAST (World Lab.), P.O. Box 8730, Beijing 100080, \\ and State Key Laboratory for Superlattices and Microstructures, Institute of Semiconductors, \\ Chinese Academy of Sciences, P.O. Box 912, Beijing 100083, China
}

\begin{abstract}
The thermal entanglement in a two-spin-qutrit system with two spins coupled by exchange interaction under a magnetic field in an arbitrary direction is investigated. Negativity, the measurement of entanglement, is calculated. We find that for any temperature the evolvement of negativity is symmetric with respect to magnetic field. The behavior of negativity is presented for four different cases. The results show that for different temperature, different magnetic field give maximum entanglement. Both the parallel and antiparallel magnetic field cases are investigated qualitatively (not quantitatively) in detail, we find that the entanglement may be enhanced under an antiparallel magnetic field.

PACS numbers: 03. 65. Ud Entanglement and quantum nonlocality (e. g. EPR paradox, Bells inequalities, GHZ states, etc.), 75.10.Jm Quantized spin models, 05. 50. +q Lattice theory and statistics (Ising, Potts, etc.), 03. 67. Lx Quantum computation
\end{abstract}

\section{INTRODUCTION}

It is well known that quantum entanglement $1,2,3$ ] plays a fundamental role in almost all efficient protocols of quantum computation (QC) and quantum information processing [4, 5]. In one proposal [6] for physical implementation of qubits, a well localized nuclear spin coupled with an electron of a donor atom in silicon plays the role of a qubit which can be individually initialized, manipulated and read out by extremely sensitive devices. In another proposal [7, 8, 9, 10], the spin of an electron in a quantum dot plays the role of a qubit. Long decoherence time and scalability to more than 100 qubits are two of the important virtues of both the schemes. In both schemes the effective interaction between the two qubits is governed by an isotropic Heisenberg Hamiltonian with Zeeman coupling of the individual spins, namely

$$
H=J S_{1} \cdot S_{2}+\gamma\left(S_{1 z}+S_{2 z}\right) .
$$

At extremely low temperatures such a qubit system may be assumed to be in its ground state. However a real physical system is always at a finite temperature and hence in a mixture of disentangled and entangled states depending on the temperature. Therefore one is naturally led to consider the thermal entanglement of such physical systems. The thermal entanglement has been extensively studied for various systems including

\footnotetext{
${ }^{*}$ Corresponding author.

†Email: gf1978zhang2001@yahoo.com
}

isotropic 11, 12, 13, 14 and anisotropic 15] Heisenberg chains, Ising model in an arbitrarily directed magnetic field [16], and cavity-QED [17 since the seminal works by Arnesen et al. 11 and Nielsen 18]. Based on the method developed in the context of quantum information, the relaxation of a quantum system towards the thermal equilibrium is investigated [19] and provides us an alternative mechanism to model the spin systems of the spin- $\frac{1}{2}$ case for the approaching of the thermal entangled states 11, 12, 13, 14, 15. It should be noted that only the uniform field case is carefully studied in the above-mentioned papers. The nonuniform case is rarely taken into account. But in any solid state construction of qubits, there is always the possibility of inhomogeneous Zeeman coupling 20, 21]. Moreover for perform quantum computing, it is necessary to control the magnetic field at each spin separately 22]. So in the theoretical analysis, the nonuniform external magnetic field should be included in the model Hamiltonian. Recently, Sun 23] and M.Asoudeh 24] investigate the thermal entanglement in the two-qubit spin model with a nonuniform magnetic field. But only the spin- $\frac{1}{2}$ case is carefully studied in the above papers. Zhang et al. 25] only consider the uniform magnetic field for spin- 1 case. In this paper, we will investigate the thermal entanglement in the two-spin-1 system with a magnetic field in an arbitrary direction. Thus we may better understand and make use of entanglement in quantum information processing through changing the environment.

Our paper is arranged as follows: first we will give the definition of negativity, the measurement of entanglement. After giving the model Hamiltonian and the so- 
lutions, we will present our calculation results by several figures. Finally, the discussion and conclusion remarks will be given.

\section{THE DEFINITION OF NEGATIVITY}

We first introduce the concept of negativity, which will be used as the entanglement measure. The PeresHorodecki criterion 26] gives a qualitative way for judging if the state is entangled. The quantitative version of the criterion was developed by Vidal and Werner 27]. They presented a measure of entanglement called negativity that can be computed efficiently, and the negativity does not increase under local manipulations of the system. The negativity of a state $\rho$ is defined as

$$
N(\rho)=\sum_{i}\left|\mu_{i}\right|,
$$

where $\mu_{i}$ is the negative eigenvalue of $\rho^{T_{1}}$, and $T_{1}$ denotes the partial transpose with respect to the first system. The negativity $N$ is related to the trace norm of $\rho^{T_{1}}$ via 27

$$
N(\rho)=\frac{\left\|\rho^{T_{1}}\right\|_{1}-1}{2} .
$$

where the trace norm of $\rho^{T_{1}}$ is equal to the sum of the absolute values of the eigenvalues of $\rho^{T_{1}}$. If $N>0$, then the two-spin state is entangled.

The state of a system at thermal equilibrium can be described by the density operator $\rho(T)=\exp (-\beta H) / Z$, where $Z=\operatorname{Tr}[\exp (-\beta H)]$ is the partition function and $\beta=1 / k_{B} T\left(k_{B}\right.$ is Boltzmann's constant being set to be unit $k_{B}=1$ hereafter for the sake of simplicity and $T$ is the temperature). The entanglement in the thermal state is called thermal entanglement.

\section{III.THE MODEL HAMILTONIAN AND THE SOLUTIONS}

The development of laser cooling and trapping provides us more ways to control the atoms in traps. Indeed, we can manipulate the atom-atom coupling constants and the atom number in each lattice well with a very good accuracy. Our system consists of two wells in the optical lattice with one spin-1 atom in each well. The lattice may be formed by three orthogonal laser beam, and we may use an effective Hamiltonian of the BoseHubbard form 28] to describe the system. The atoms in the Mott regime make sure that each well contains only one atom. For finite but small hopping term $t$, we can expand the Hamiltonian into powers of $t$ and get [29],

$$
H=\epsilon+J\left(S_{1} \cdot S_{2}\right)+K\left(S_{1} \cdot S_{2}\right)^{2},
$$

where $J=-\frac{2 t^{2}}{U_{2}}, K=-\frac{2 t^{2}}{3 U_{2}}-\frac{4 t^{2}}{U_{0}}$ with $t$ the hopping matrix elements, and $\epsilon=J-K$. $U_{s}(s=0,2)$ represents the Hubbard repulsion potential with total spin $s$, a potential $U_{s}$ with $s=1$ is not allowed due to the identity of the bosons with one orbital state per well, Since term $\epsilon$ contains no interaction, we can ignore it in the following discussions and it would not change the thermal entanglement. For simplification, $J \gg K$ is assumed and the nonlinear couplings is ignored. So the Hamiltonian Eq.(4) becomes

$$
H=J\left(S_{1} \cdot S_{2}\right)
$$

with an nonuniform external magnetic field in an arbitrary direction, our system is described by

$$
H=J\left(S_{1 x} S_{2 x}+S_{1 y} S_{2 y}\right)+B \cos [\theta] S_{1 z}+B \sin [\theta] S_{2 z} .
$$

in which the neglected exchange coupling term along the $z$-axes is assumed to be much smaller than the coupling in the x-y plane. Where $S_{\alpha}(\alpha=x, y, z)$ are the spin operator, $J$ is the strength of Heisenberg interaction and the magnetic field is assumed to be along the $z$-axes. When the total spin for each site $S_{j}=1(j=1,2)$, its components take the form,

$$
\begin{aligned}
S_{j x} & =\frac{1}{\sqrt{2}}\left(\begin{array}{lll}
0 & 1 & 0 \\
1 & 0 & 1 \\
0 & 1 & 0
\end{array}\right), \\
S_{j y} & =\frac{1}{\sqrt{2}}\left(\begin{array}{lll}
0 & -i & 0 \\
i & 0 & -i \\
0 & i & 0
\end{array}\right), \\
S_{j z} & =\left(\begin{array}{lll}
1 & 0 & 0 \\
0 & 0 & 0 \\
0 & 0 & -1
\end{array}\right)
\end{aligned}
$$

In the following calculation we will select $J$ as the energy unit and set $J=1$.

To evaluate the thermal entanglement we first of all find the eigenvalues and the corresponding eigenstates of the Hamiltonian Eq.(6). which are seen to be

$$
\begin{aligned}
H\left|\psi_{1}\right\rangle & =0, H\left|\psi_{2}\right\rangle=-B_{+}\left|\psi_{2}\right\rangle \\
H\left|\psi_{3}\right\rangle & =B_{+}\left|\psi_{3}\right\rangle, H\left|\psi_{4}^{ \pm}\right\rangle=-m_{\mp}\left|\psi_{4}^{ \pm}\right\rangle \\
H\left|\psi_{5}^{ \pm}\right\rangle & =m_{ \pm}\left|\psi_{5}^{ \pm}\right\rangle, H\left|\psi_{6}^{ \pm}\right\rangle= \pm \xi\left|\psi_{6}^{ \pm}\right\rangle .
\end{aligned}
$$

where $B_{ \pm}=B \cos [\theta] \pm B \sin [\theta], \xi=\sqrt{2+B_{-}^{2}}, \zeta=$ $\sqrt{4+B_{-}^{2}}, m_{ \pm}=\frac{1}{2}\left(B_{+} \pm \zeta\right)$.

And the corresponding eigenstates are explicitly given 
by

$$
\begin{aligned}
\left|\psi_{1}\right\rangle & =\frac{1}{\xi}\left(|-1,1\rangle+B_{-}|0,0\rangle-|1,-1\rangle\right), \\
\left|\psi_{2}\right\rangle & =|-1,-1\rangle \\
\left|\psi_{3}\right\rangle & =|1,1\rangle \\
\left|\psi_{4}^{ \pm}\right\rangle & =\frac{1}{\sqrt{1+S_{ \pm}^{2}}}\left(|-1,0\rangle+S_{ \pm}|0,-1\rangle\right), \\
\left|\psi_{5}^{ \pm}\right\rangle & =\frac{1}{\sqrt{1+S_{ \pm}^{2}}}\left(|0,1\rangle+S_{ \pm}|1,0\rangle\right),
\end{aligned}
$$

$$
\left|\psi_{6}^{ \pm}\right\rangle=\frac{1}{\sqrt{1+R_{ \pm}^{2}+\left(1 \pm B_{-} R_{ \pm}\right)^{2}}}\left[|-1,1\rangle \pm R_{ \pm}|0,0\rangle+\left(1 \pm B_{-} R_{ \pm}\right)|1,-1\rangle\right]
$$

where $R_{ \pm}= \pm B_{-}+\xi$ and $S_{ \pm}=\frac{1}{2}\left(B_{-} \pm \zeta\right)$. Here $|x, y\rangle$ $(x=1,0,-1$ and $y=1,0,-1)$ are the eigenstates of $S_{1 z} S_{2 z}$. The density operator $\rho$ can be expressed in terms of the eigenstates and the corresponding eigenvalues as

$$
\rho=\frac{1}{Z} \sum \exp \left[-\beta E_{l}\right]\left|\Psi_{l}\right\rangle\left\langle\Psi_{l}\right|,
$$

where $E_{l}$ is the eigenvalue of the corresponding eigen- states and the partition function is $Z=1+2 \cosh [\beta \xi]+$ $4 \cosh \left[\frac{1}{2} \beta \zeta\right] \cosh \left[\frac{\beta B_{+}}{2}\right]+2 \cosh \left[\beta B_{+}\right]$.

For our purpose to evaluate the negativity in what following we need to have a partially transposed density matrix $\rho^{T_{1}}$ of original density matrix $\rho$ with respect to the eigenbase of any one spin particle ( say particle 1 ) which is found in the basis $|x, y\rangle(x=1,0,-1$ and $y=1,0,-1)$ as

$$
\rho^{T_{1}}=\frac{1}{Z}\left(\begin{array}{ccccccccc}
e^{-\beta B_{+}} & 0 & 0 & 0 & q_{+} & 0 & 0 & 0 & \frac{-1+\cosh [\beta \xi]}{\xi^{2}} \\
0 & M e^{-\frac{\beta B_{+}}{2}} & 0 & 0 & 0 & u_{-} & 0 & 0 & 0 \\
0 & 0 & W_{-} & 0 & 0 & 0 & 0 & 0 & 0 \\
0 & 0 & 0 & Q_{-} & 0 & 0 & 0 & u_{+} & 0 \\
q_{+} & 0 & 0 & 0 & 1+2\left(\frac{-1+\cosh [\beta \xi]}{\xi^{2}}\right) & 0 & 0 & 0 & q_{-} \\
0 & u_{-} & 0 & 0 & 0 & M e^{\frac{\beta B_{+}}{2}} & 0 & 0 & 0 \\
0 & 0 & 0 & 0 & 0 & 0 & W_{+} & 0 & 0 \\
0 & 0 & 0 & u_{+} & 0 & 0 & 0 & Q_{+} & 0 \\
\frac{-1+\cosh [\beta \xi]}{\xi^{2}} & 0 & 0 & 0 & q_{-} & 0 & 0 & 0 & e^{\beta B_{+}}
\end{array}\right)
$$

where $M=\cosh \left[\frac{1}{2} \beta \zeta\right]-\frac{\sinh \left[\frac{1}{2} \beta \zeta\right] B_{-}}{\zeta}, q_{ \pm}=-\frac{1}{\zeta}\left[e^{-\frac{1}{2}} \beta(\zeta \pm\right.$ $\left.\left.B_{+}\right)\left(-1+e^{\beta \zeta}\right)\right], u_{ \pm}=\frac{ \pm B_{-}(1-\cosh [\beta \xi])-\xi \sinh [\beta \xi]}{\xi^{2}}, W_{ \pm}=$ $\frac{1}{\xi^{2}}\left(1+\cosh [\beta \xi]\left(1+B_{-}^{2}\right) \pm \xi B_{-} \sinh [\beta \xi]\right), \quad Q_{ \pm}=$ $\frac{1}{2} e^{\frac{1}{2} \beta\left(\zeta \pm B_{+}\right)}\left(1+\frac{B_{-}}{\zeta}\right)+\frac{2 e^{ \pm \frac{1}{2} \beta\left(\mp \zeta+B_{+}\right)}}{4+B_{-}\left(B_{-}+\zeta\right)}$.

We perform the numerical diagonalization of the density matrix and the numerical results of the entanglement measure $N$ is presented. In Fig.1, we give the contour of negativity for different temperature with respect to $B$ and $\theta$. From Fig.1, we can see that the evolvement of negativity is symmetric with respect to magnetic field. The maximum negativity arrives at the point $B=0$ when $T=0.05$ and $T=0.2$. With the increasing temperature, the area of $B$ at which the system can reach maximum negativity becomes narrower and even arrives zero for a higher temperature (for example $T=0.6$ and $T=1.2$ ). When the temperature is low, only one peak appears. For $T=0.6$ and $T=1.2$, the double peak structure 

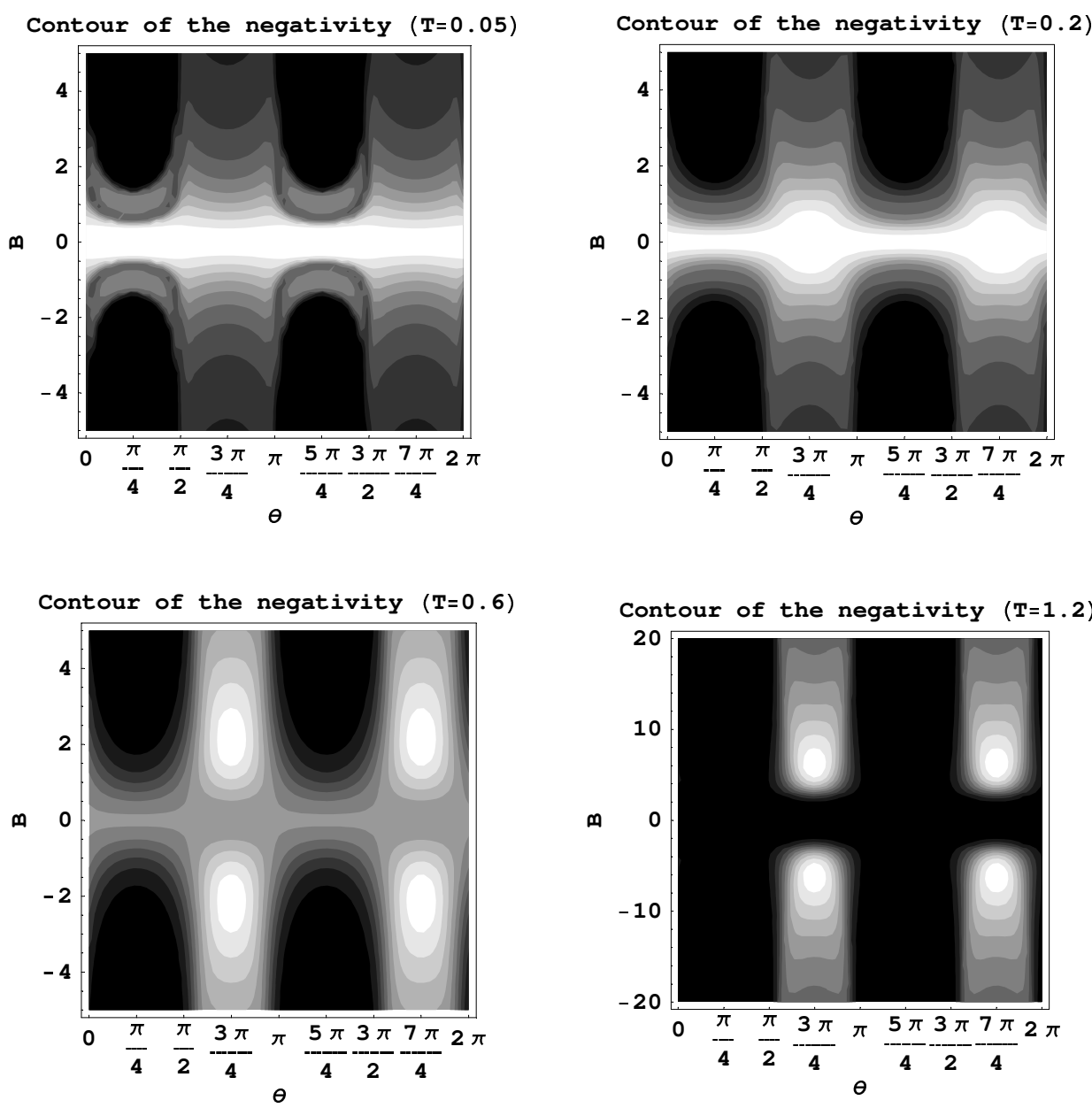

FIG. 1: The contour of negativity for different temperature vs. $B$ and $\theta$. The brighter place means the higher negativity.
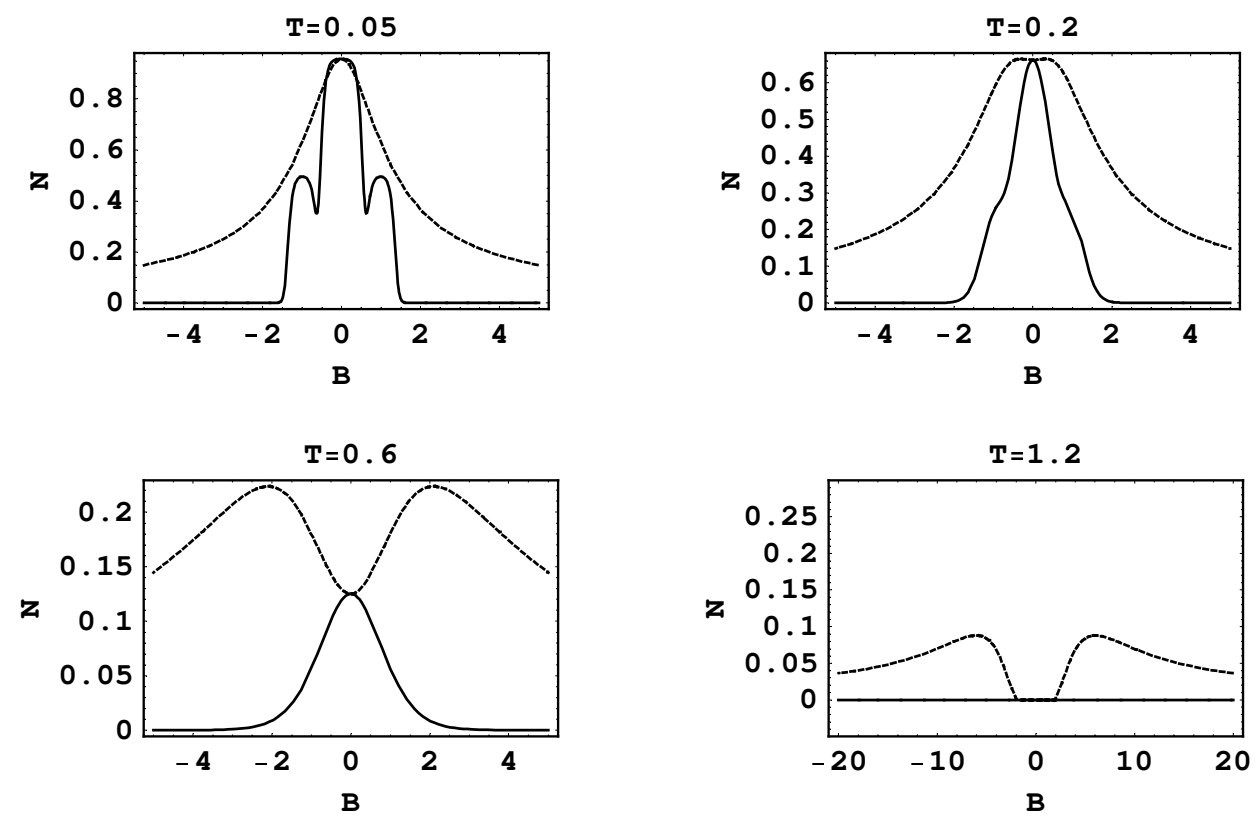

FIG. 2: The negativity versus $B$ for different temperature. solid curve for $\theta=\frac{\pi}{4}$ and dotted curve for $\theta=\frac{3 \pi}{4}$. 
takes place. We can also find that the evolvement of negativity is periodic with respect to the polar angle $\theta$ and the double peak structure takes place at $\theta=\left(n+\frac{3}{4}\right) \pi$, $(n=0,1, \ldots)$. From these figures, we know that the negativity gets smaller with the increasing magnetic field amplitude. For a higher temperature (say $T=1.2$ ), we can see that $N$ arrives zero near $B=0$. But $N$ increases with the value of $B$ to a peak value for $\theta=\left(n+\frac{3}{4}\right) \pi$ cases, after this peak, $N$ will decrease monotonously.

In order to see clearly the change of the negativity, we give the results for $\theta=\frac{\pi}{4}$ and $\theta=\frac{3 \pi}{4}$. This corresponds to the parallel and antiparallel magnetic field case. We give our calculation results in Fig.2 (solid line for $\theta=\frac{\pi}{4}$, dotted line for $\theta=\frac{3 \pi}{4}$ ), in which the negativity is plotted in the whole parameter space at a given temperature, and four typical cases are shown. As the temperature is low $(T=0.05)$, we may find that there are two features. First, there are three sharp peaks (different from the spin$\frac{1}{2}$ case for which only one peak appears, the results for spin- $\frac{1}{2}$ case can be seen from Ref [23]) and the center of the middle one locates at $B=0$, where the negativity is about 1 . As we increase the external field $B, N$ rapidly decays. When $T=0.6$, we can see that the three peaks evolve into one. That is to say, the left and the right peak disappear, the middle peak gets shorter as we increase the temperature. As the temperature is further increased, for example $T=1.2$, the entanglement is entirely destroyed.

Compared with the parallel magnetic field case $(\theta=$ $\left.\frac{\pi}{4}\right)$, when at the low temperature, for antiparallel magnetic field $\left(\theta=\frac{3 \pi}{4}\right)$ case, there is no three-peaks structure emerges and only a peak that monotonously decreases with the value of $B$. But the negativity decreases more slowly than in the parallel case which means that in the strong field region, the parallel field and the antiparallel field demonstrate obviously different effects on the entanglement. We can find that in all parameter space, the negativity of the magnetic field with antiparallel direction is much larger than that of parallel field. These results can be seen in these figures. As the temperature increase $(T=0.6)$, the feature of $N$ will be changed. The primary peak at $T=0.05$ splits into two peaks. For the parallel field case, the maximum $N$ appears at $B=0$, but for antiparallel field case, at the point $N$ is a minimum point. The results is the same with the one which can be found in Ref [23]. For $T=0.6$, if we apply an antiparallel field, $N$ will be enhanced more than two times. This again illuminates the fact that the well-chosen external field can partially weaken the destructive effect of thermal fluctuation and enhance the entanglement. In other words, for a certain temperature, a well-chosen external field is helpful for entanglement. At a higher temperature $(T=1.2)$, we can see that $N$ arrives zero at $B=0$ for the both case. But $N$ increases with the value of $B$ to a peak value for the antiparallel case, after this peak, $N$ will decrease monotonously.

\section{CONCLUSIONS}

We investigated qualitatively (not quantitatively) the effects of a magnetic field in an arbitrary direction on the thermal entanglement in the two-spin-1 system in terms of the measure of entanglement called "negativity". We give results for different temperatures. We find that the temperature and the magnetic field can affect the feature of the thermal entanglement significantly. At a certain temperature, the antiparallel magnetic field is helpful for entanglement. In other words, the entanglement may be enhanced under an antiparallel magnetic field.

[1] A. Einstein, B. Podolsky and N. Rozen, Phys. Rev., 47, 777 (1935).

[2] E. Schrdinger, Naturwiss. 23, 807 (1935).

[3] J. S. Bell, Physics 1, 195 (1964).

[4] C. H. Bennett and D. P. Divincenzo, Nature, 407, 247 (2000).

[5] M. A. Nielson and I. L. Chuang, Quantum Computation and Quantum Information, (Cambrdige University Press, Cambrdige, 2000).

[6] B. E. Kane, Nature, 393133 (1998).

[7] D. Loss and D. P. DiVincenzo, Phys. Rev. A 57, 120(1998); J. Levy, Phys. Rev. A 64, 052306 (2001).

[8] DiVincenzo et al, Nature, Vol. 408, p. 339-342 (2000).

[9] G. Burkard and G. Loss, Phys. Rev. Lett. 88, 047903 (2002).

[10] A. Imamoglu et al, Phys. Rev. Lett. 83, 4204 (1999).

[11] M. C. Arnesen, S. Bose and V. Vedral, Phys. Rev. Lett. 87, 017901 (2001).

[12] K. M. OConnor and W. K. Wootters, Phys. Rev. A 63,052302 (2001).

[13] X. Wang, Phys. Rev. A 66, 044305 (2002); X. Wang, Phys. Rev. A 66, 034302 (2002).

[14] G. F. Zhang, J. Q. Liang, Q. W. Yan, Chin. Phys. Lett. 20, 452(2003).

[15] X. Wang, Phys. Rev. A 64, 012313 (2001); G. Lagmago Kamta and A. F. Starace, Phys. Rev. Lett. 88, 107901(2002).

[16] D. Gunlycke V. M. Kendon, and V. Vedral, Phys. Rev. A 64, 042302 (2001).

[17] S. Mancini, and S. Bose, e-print quant-ph/0111055

[18] M. A. Nielsen, e-print quant-ph/0011036

[19] V. Scarani et al., Phys. Rev. Lett. 88, 097905 (2002).

[20] X. Hu, and S. Das Sarma, Phys. Rev. A. 61, 062301(2000).

[21] X. Hu, R. de Sousa and S. Das Sarma, Phys. Rev. Lett. 86, 918(2001).

[22] Y. Makhlin, G. Schön and A. Shnirman, Rev. Mod. Phys. 73, 357 (2001)

[23] Y. Sun, Y. Chen and H. Chen, Phys. Rev. A. 68, 044301(2003).

[24] M. Asoudeh and V. Karimipour, e-print quant-ph/0407073

[25] G. F. Zhang, J. Q. Liang, G. E. Zhang and Q. W. Yan, Eur. Phys. J. D 32, 409-412 (2005).

[26] A. Peres, Phys. Rev. Lett. 77, 1413 (1996); M. 
Horodecki,P. Horodecki, and R. Horodecki, Phys. Lett. A 223, 1(1996).

[27] G. Vidal and R. F. Werner, Phys. Rev. A 65, 032314(2002).
[28] D. Jaksch, C. Bruder, J. I. Cirac, C. W. Gardiner and P. Zoller, Phys. Rev. Lett. 81, 3108(2003).

[29] S. K. Yip, Phys. Rev. Lett. 90, 250402(1998). 